BULL. AUSTRAL. MATH. SOC.

VOL. 14 (1976), 129-136.

\title{
On the complex nonlinear complementary problem
}

\section{J. Parida and B. Sahoo}

The complex nonlinear complementarity problem considered here is the following: find $z$ such that

$$
\begin{gathered}
g(z) \in S^{*}, z \in S, \\
\operatorname{Re}(g(z), z)=0,
\end{gathered}
$$

where $S$ is a polyhedral cone in $c^{n}, S^{*}$ the polar cone, and $g$ is a mapping from $c^{n}$ into itself. We study the extent to which the existence of a $z \in S$ with $g(z) \in S^{*}$ (feasible point) implies the existence of a solution to the nonlinear complementarity problem, and extend, to nonlinear mappings, known results in the linear complementarity problem on positive semidefinite matrices.

\section{Introduction}

Given $g: c^{n} \rightarrow c^{n}$, the nonlinear complementarity problem consists of finding a $z$ such that

$$
\begin{gathered}
g(z) \in S^{*}, \quad z \in S, \\
\operatorname{Re}(g(z), z)=0,
\end{gathered}
$$

where $S$ is a polyhedral cone in $C^{n}$ and $S^{*}$ the polar cone of $S$.

Problems of the form (1.1), where $g(z)$ is the affine transformation $M z+q$, have already appeared in the literature. McCallum [4] showed that

Received 12 November 1975. 
when $M \in C^{n \times n}$ is positive semi-definite, $S$ and $S^{*}$ are sectors in complex space, and the constraints are feasible; then a solution exists to the corresponding linear complementarity problem. Mond [5] extended this result to the more general complex linear complementarity problem, where the constraints are restricted to polyhedral cones.

In this paper, we have studied the existence of a solution to (1.1) under feasibility assumptions. Theorems analogous to those proved by McCall um [4] and Mond [5] in the complex linear case, and Moré [6] and cottle [2] in the real case are obtained by considering monotone functions. These mappings are nonlinear versions of positive semi-definite matrices.

\section{Notations and preliminaries}

Denote by $C^{n}\left[R^{n}\right] \quad n$-dimensional complex [real] space; denote by $c^{m \times n}\left[R^{m \times n}\right]$ the vector space of all $m \times n$ complex [real] matrices; denote by $R_{+}^{n}=\left\{x \in R^{n}: x_{i} \geq 0,1 \leq i \leq n\right\}$ the non-negative orthant of $R^{n}$; and for any $x, y \in R^{n}, x \geq y$ denotes $x-y \in R_{+}^{n}$. If $A$ is a complex matrix or vector, then $A^{T}, \bar{A}$, and $A^{H}$ denote its transpose, complex conjugate, and conjugate transpose. For $x, y \in C^{n},(x, y) \equiv y^{H} x$ denotes the inner product of $x$ and $y$.

A nonempty set $S \subset C^{n}$ is a polyhedral cone if for some positive integer $k$ and $A \in C^{n \times k}$,

$$
S=\left\{A x: x \in R_{+}^{k}\right\} .
$$

The polar of $S$ is the cone $S^{*}$ defined by

$$
S^{*}=\left\{y \in C^{n}: x \in S \Rightarrow \operatorname{Re}(x, y) \geq 0\right\},
$$

or equivalently by

$$
S^{*}=\left\{y \in C^{n}: \operatorname{Re}\left(A^{H} y\right) \geq 0\right\} \text {. }
$$

The interior of $S^{*}$, int $S^{*}$, is given by

$$
\text { int } S^{*}=\left\{y \in S^{*}: \operatorname{Re}\left(A^{H} y\right)>0\right\} \text {. }
$$


A mapping $g: c^{n}+C^{n}$ is said to be monotone on $S$ if $\operatorname{Re}\left(g\left(z^{1}\right)-g\left(z^{2}\right), z^{1}-z^{2}\right) \geq 0$ for each $z^{1}, z^{2} \in S$, and strictly monotone if the strict inequality holds whenever $z^{l} \neq z^{2}$.

We shall make use of the following definition of convexity [1] of a complex-valued function with respect to a cone.

A mapping $g: C^{n} \rightarrow C^{n}$ is concave with respect to the polyhedral cone $S$ if, for all $z^{1}, z^{2} \in C^{n}$ and for all $\lambda \in[0,1]$,

$$
g\left(\lambda z^{1}+(1-\lambda) z^{2}\right)-\lambda g\left(z^{1}\right)-(1-\lambda) g\left(z^{2}\right) \in S \text {. }
$$

Given a mapping $g: C^{n} \rightarrow C^{n}$, $\operatorname{Re} z^{H} g(z)$ is convex with respect to $R_{+}$if, for all $z^{1}, z^{2} \in C^{n}$ and $\lambda \in[0,1]$, $\lambda \operatorname{Re}\left(g\left(z^{1}\right), z^{1}\right)+(1-\lambda) \operatorname{Re}\left(g\left(z^{2}\right), z^{2}\right)$

$-\operatorname{Re}\left(g\left(\lambda z^{1}+(1-\lambda) z^{2}\right), \lambda z^{1}+(1-\lambda) z^{2}\right) \geq 0$.

\section{Solutions of variational inequalities}

Hartman and Stampacchia [3] have proved the following result on variational inequalities: if $F: R^{n} \rightarrow R^{n}$ is a continuous mapping on the nonempty, compact, convex set $K \subset R^{n}$, then there is an $x^{0}$ in $K$ such that

$$
\left(F\left(x^{0}\right), x-x^{0}\right) \geq 0
$$

for all $x \in K$. Since $C^{n}$ can be identified with $R^{2 n}$, a natural extension of this result to complex space can be obtained as follows.

THEOREM 3.1. If $g: c^{n} \rightarrow c^{n}$ is a continuous mapping on the nonempty, compact, convex set $S \subset C^{n}$, then there is a $z^{0}$ in $S$ with

$$
\operatorname{Re}\left(g\left(z^{0}\right), z-z^{0}\right) \geq 0
$$

for all $z \in S$.

A polyhedral cone is a closed, convex set, but not bounded. We shall show that Theorem 3.1 holds for polyhedral cones under a very weak 
restriction on the growth of the mapping $g$.

Let $S$ be a polyhedral cone in $C^{n}$. Then there is a positive integer $k$ and a matrix $A \in C^{n \times k}$ such that $S=\left\{A x: x \in R_{+}^{k}\right\}$. For a constant $p>0$, we denote $z(p)=\left\{A x: x_{i}=p, 1 \leq i \leq k\right\}$ and for any $z=A x \in S$, we write $z \leq z(p)$ if $\|x\|_{\infty} \leq p$, where $\|x\|_{\infty}=\max \left\{\left|x_{i}\right|: 1 \leq i \leq k\right\}$.

LEMMA 3.2. Let $y^{0} \in C^{n}$ be given, and assume $S$ is a polyhedral cone in $C^{n}$. Then an element $z^{0} \in S$ satisfies

$$
\operatorname{Re}\left(y^{0}, z-z^{0}\right) \geq 0
$$

for all $z \in S$ provided there is a vector $z(p)>z^{0}$ in $S$ such that (3.3) holds for all

$$
z \in S_{p}=\{z \in S: z \leq z(p)\}
$$

Proof. Let $z \in S$, and write $u=\lambda z+(1-\lambda) z^{0}$ for $0<\lambda<1$. Since $S$ is a polyhedral cone, $u \in S$, and also it follows that there exist $A \in C^{n \times k}$ and $x, x^{0} \in R_{+}^{k}$ such that $z=A x$ and $z^{0}=A x^{0}$. Then $u \in S_{p}$ if $\left\|\lambda x+(1-\lambda) x^{0}\right\|_{\infty} \leq p$. Since $\left\|x^{0}\right\|_{\infty}<p$, we can choose $\lambda$ sufficiently small so that $u$ lies in $S_{p}$. Then

$$
0 \leq \operatorname{Re}\left(y^{0}, u-z^{0}\right)=\lambda \operatorname{Re}\left(y^{0}, z-z^{0}\right),
$$

and consequently, $z^{0}$ satisfies (3.3) for all $z \in S$.

THEOREM 3.3. Let $g: C^{n} \rightarrow C^{n}$ be a continuous mapping on the polyhedral cone $S$. If there are vectors $z(p), u \in S$, with $z(p)>u$ such that $\operatorname{Re}(g(z), z-u) \geq 0$ for all $z=z(p)$ in $S$, then there is a $z^{0} \leq z(p)$ in $S$ with

$$
\operatorname{Re}\left(g\left(z^{0}\right), z-z^{0}\right) \geq 0
$$

for azz $z \in S$.

Proof. Consider the set $S_{p}=\{z \in S: z \leq z(p)\}$. Since $S$ is a 
polyhedral cone, we can write $S_{p}=\left\{A x: x \in R_{+}^{k},\|x\|_{\infty} \leq p\right\}$ which is obviously a compact, convex set in $c^{n}$. Therefore by Theorem 3.1 , there is a $z^{0} \in S_{p}$ satisfying (3.4) for all $z \in S_{p}$. If $z^{0}<z(p)$, then taking $y^{0}=g\left(z^{0}\right)$ in Lemma 3.2, we get the desired result. If $z^{0}=z(p)$, then by the hypothesis, $\operatorname{Re}\left(g\left(z^{0}\right), z^{0}-u\right) \geq 0$. Since $\operatorname{Re}\left(g\left(z^{0}\right), z-z^{0}\right) \geq 0$ for all $z \in S_{p}$, it follows that $\operatorname{Re}\left(g\left(z^{0}\right), z-u\right) \geq 0$ for all $z \in S_{p}$. But $u<z(p)$, and thus by Lemma 3.2, $\operatorname{Re}\left(g\left(z^{0}\right), z-u\right) \geq 0$ for all $z \in S$. Also $u \in S_{p}$, and so $\operatorname{Re}\left(g\left(z^{0}\right), u-z^{0}\right) \geq 0$. Now adding the last two inequalities, we obtain $\operatorname{Re}\left(g\left(z^{0}\right), z-z^{0}\right) \geq 0$ for all $z \in S$.

\section{Solvability of the complementarity problem}

We now prove a lemma which gives the connection between variational inequalities discussed in section 3 and the nonlinear complementarity problem (1.1).

LEMMA 4.1. Let $S$ be a polyhedral cone in $c^{n}$, and let $g: c^{n} \rightarrow c^{n}$ be continuous on $S$. If there is a $z^{0} \in S$ such that

$$
\operatorname{Re}\left(g\left(z^{0}\right), z-z^{0}\right) \geq 0
$$

for alz $z \in S$, then

$$
g\left(z^{0}\right) \in S^{*} \text { and } \operatorname{Re}\left(g\left(z^{0}\right), z^{0}\right)=0 \text {. }
$$

Thus $z^{0}$ is a solution to (1.1).

Proof. If $\operatorname{Re}\left(g\left(z^{0}\right), z-z^{0}\right) \geq 0$ for all $z \in S$, then $\operatorname{Re}\left(g\left(z^{0}\right), z\right) \geq \operatorname{Re}\left(g\left(z^{0}\right), z^{0}\right)$ for all $z \in S$. Since $S$ is a polyhedral cone, $z+z^{0} \in S$ for all $z \in S$. Then $\operatorname{Re}\left(g\left(z^{0}\right), z+z^{0}\right) \geq \operatorname{Re}\left(g\left(z^{0}\right), z^{0}\right)$ for all $z \in S$ and consequently, $\operatorname{Re}\left(g\left(z^{0}\right), z\right) \geq 0$ for all $z \in S$ and, in particular, $\operatorname{Re}\left(g\left(z^{0}\right), z^{0}\right) \geq 0$. So $g\left(z^{0}\right) \in S^{*}$. Since $0 \in S$, from 
(4.1) we get $\operatorname{Re}\left(g\left(z^{0}\right), z^{0}\right) \leq 0$, and hence $\operatorname{Re}\left(g\left(z^{0}\right), z^{0}\right)=0$.

THEOREM 4.2. Let $g: c^{n}+c^{n}$ be a continuous monotone function on $S$, a polynedral cone in $c^{n}$. If there is a $u \in S$ with $g(u) \in$ int $S^{*}$, then (1.1) has a solution $z^{0} \in S$.

Proof. Since $g(z)$ is monotone on $S$,

$$
\operatorname{Re}(g(z), z-u) \geq \operatorname{Re}(g(u), z-u) .
$$

If $z=A x, u=A y, x, y \in R_{+}^{k}$, then

$$
\operatorname{Re}(g(u), z-u)=(x-y)^{T} \operatorname{Re}\left(A^{H} g(u)\right) .
$$

Since $g(u) \in$ int $S^{*}, \operatorname{Re}\left(A^{H} g(u)\right)>0$. It is then clear that there is a vector $z(p)>u$ in $S$ such that $(x-y)^{T} \operatorname{Re}\left(A^{H} g(u)\right) \geq 0$ for all $z=z(p)$ in $S$. Theorem 3.3 with Lemma 4.1 now gives the result.

REMARKS 4.3. $M \in C^{n \times n}$ is said to be positive semi-definite if $\operatorname{Re} z^{H} M z \geq 0$ for all $z \in C^{n}$. If $g(z)$ is defined by $g(z)=M z+q$ for some matrix $M$ and $q$ in $C^{n}$, then $g$ is monotone on $S$ if $M$ is positive semi-definite. Thus Theorem 4.2 is a generalization to nonlinear mappings of the results of McCallum [4, Theorem 4.5.1] and Mond [5, Theorem 5] in the complex linear complementarity problem on positive semidefinite matrices.

Recently, Moré [6] has extended the result of Cottle [2] on linear complementarity. problem in real space to nonlinear mappings. If $S=R_{+}^{n}$ and $g: R^{n} \rightarrow R^{n}$ is a continuous mapping on $R_{+}^{n}$, then Theorem 4.2 reduces to the result of Moré [6, Theorem 3.2].

If $g$ is strictly monotone on $S$, then there is at most one $z^{0} \in S$ which satisfies (1.1). For if $z^{0}$ and $w^{0}$ are two solutions, then $\operatorname{Re}\left(g\left(z^{0}\right)-g\left(w^{0}\right), z^{0}-w^{0}\right)=-\operatorname{Re}\left(g\left(z^{0}\right), w^{0}\right)-\operatorname{Re}\left(g\left(w^{0}\right), z^{0}\right) \leq 0$, and consequently, $z^{0}=w^{0}$.

LEMMA 4.4. Let $S$ be a polyhedral cone in $c^{n}$. If $g: c^{n} \rightarrow c^{n}$ 
is a continuous function concave with respect to $S^{*}$ and $\operatorname{Re} z^{H} g(z)$ is convex with respect to $R_{+}$, then $g(z)$ is monotone on $S$.

Proof. Concavity of $g(z)$ with respect to $S^{*}$ and $z^{1}, z^{2} \in S$ imply that for $\lambda \in(0,1)$,

(4.2) $\operatorname{Re}\left(g\left(\lambda z^{1}+(1-\lambda) z^{2}\right), \lambda z^{1}+(1-\lambda) z^{2}\right)$

$$
-\operatorname{Re}\left(\lambda g\left(z^{1}\right)+(1-\lambda) g\left(z^{2}\right), \lambda z^{1}+(1-\lambda) z^{2}\right) \geq 0 \text {. }
$$

Convexity of $\operatorname{Re} z^{H} g(z)$ gives

(4.3) $\lambda \operatorname{Re}\left(g\left(z^{1}\right), z^{1}\right)+(1-\lambda) \operatorname{Re}\left(g\left(z^{2}\right), z^{2}\right)$

$$
-\operatorname{Re}\left(g\left(\lambda z^{1}+(1-\lambda) z^{2}\right), \lambda z^{1}+(1-\lambda) z^{2}\right) \geq 0 .
$$

From (4.2) and (4.3),

$$
\lambda(1-\lambda) \operatorname{Re}\left(g\left(z^{1}\right)-g\left(z^{2}\right), z^{1}-z^{2}\right) \geq 0,
$$

and consequently, $g(z)$ is monotone on $S$.

Now we are able to give a different version of Theorem 4.2.

THEOREM 4.5. Let $g: c^{n} \rightarrow c^{n}$ be continuous on $S$ and concave with respect to $S^{*}$ on $C^{n}$. Let $\operatorname{Re} z^{H} g(z)$ be convex with respect to $R_{+}$on $c^{n}$. If there is a $u \in S$ with $g(u) \in$ int $S^{*}$, then (1.1) has a solution $z^{0}$ in $S$.

REMARKS 4.6. It is proved by the first author [7] that if $g$, in addition to satisfying the hypotheses of Theorem 4.5, is analytic, then the nonlinear program

$$
\text { (P): } \begin{array}{ll}
\text { minimize } \operatorname{Re} z^{H} g(z) \\
\text { subject to } g(z) \in S^{*}, z \in S,
\end{array}
$$

is a self-dual problem with zero optimal value. Thus an optimal point of (P) under the said restrictions on the growth of $g$ is a solution to (1.1).

Moreover, any feasible solution to (P) which makes the objective function vanish is necessarily a solution to (1.1). So a critical study of (P) may shed more light on this problem of existence of a solution to (1.1) 
under feasibility assumptions.

\section{References}

[1] Robert A. Abrams, "Nonlinear programming in complex space: sufficient conditions and duality", J. Math. Anaz. Appl. 38 (1972), 619-632.

[2] Richard W. Cottle, "Note on a fundamental theorem in quadratic programming", J. Soc. Indust. App 2. Math. 12 (1964), 663-665.

[3] P. Hartman and G. Stampacchia, "On some nonlinear elliptic different differential functional equations", Acta Math. 115 (1966), $271-310$.

[4] Charles J. MCCallum, Jr, "Existence theory for the complex linear complementarity problem", J. Math. Anal. Appl. 40 (1972), 738-762.

[5] Bertram Mond, "On the complex complementarity problem", Bull. Austral. Math. Soc. 9 (1973), 249-257.

[6] Jorge J. Moré, "Classes of functions and feasibility conditions in nonlinear complementarity problems", Math. Programming 6 (1974), $327-338$.

[7] J. Parida, "Self-duality in complex mathematical programming", Cahiers Centre Etudes Recherche Opér. (to appear).

Department of Mathematics,

Regional Engineering College,

Rourkela,

Orissa,

India. 